\title{
Variational approach to the sixth-order boundary value problems
}

\author{
Ji-Huan $\mathrm{He}$ \\ LNM, Institute of Mechanics, Chinese Academy of Sciences, Beijing 10080, PR China \\ College of Science, Shanghai Donghua University, P.O. Box 471, 1882 Yan'an Xilu Road, \\ Shanghai 200051, China \\ Institute of Applied Mathematics and Mechanics, 149 Yanchang Road, Shanghai 200072, China
}

\begin{abstract}
Recently, Wazwaz [Appl. Math. Comput. 118 (2001) 311-325] applied the Adomian's decomposition method to solve analytically the solution of sixth-order boundary value problems. The same problem is discussed via the variational principle, which reveals to be much more simpler and much more efficient.

(c) 2002 Elsevier Science Inc. All rights reserved.
\end{abstract}

Keywords: Boundary value problem; Variational principle; Ritz method

The sixth-order boundary value problems,

$$
y^{(\mathrm{vi})}(x)=f(x, y), \quad 0<x<b,
$$

with proper boundary conditions were discussed by Wazwaz [1] using the Adomian decomposition method [1,2]. A much simpler solution is obtained using the Ritz's method which is based on variational theory.

By the semi-inverse method [3], the variational principle of the above equation is easily obtained, which reads

$$
J(y)=\int_{0}^{b}\left\{\frac{1}{2}\left(y^{\prime \prime \prime}\right)^{2}+F(x, y)\right\} \mathrm{d} x,
$$

where $F$ is the potential function defined as

E-mail address: jhhe@dhu.edu.cn (J.-H. He).

0096-3003/02/\$ - see front matter (c) 2002 Elsevier Science Inc. All rights reserved. doi:10.1016/S0096-3003(02)00381-8 


$$
\frac{\partial F}{\partial y}=f .
$$

To compare with Wazwaz's method, we consider the same example as discussed in Ref. [1]:

$$
y^{(\mathrm{vi})}(x)=-6 \mathrm{e}^{x}+y(x), \quad 0<x<1,
$$

subject to the boundary conditions

$$
\begin{aligned}
& y(0)=1, \quad y^{\prime \prime}(0)=-1, \quad y^{(\mathrm{iv})}(0)=-3, \\
& y(1)=0, \quad y^{\prime \prime}(1)=-2 e, \quad y^{(\mathrm{iv})}(0)=-4 e .
\end{aligned}
$$

Its variational principle reads

$$
J(y)=\int_{0}^{1}\left\{\frac{1}{2}\left(y^{\prime \prime \prime}\right)^{2}-6 \mathrm{e}^{x} y+\frac{1}{2} y^{2}\right\} \mathrm{d} x .
$$

Applying Ritz's method, we choose a trial function satisfying all the boundary conditions.

$$
\begin{aligned}
y & =(1-x)\left(a_{0}+a_{1} x+a_{2} x^{2}+a_{3} x^{3}+a_{4} x^{4}\right) \\
& =a_{0}+\left(a_{1}-a_{0}\right) x+\left(a_{2}-a_{1}\right) x^{2}+\left(a_{3}-a_{2}\right) x^{3}+\left(a_{4}-a_{3}\right) x^{4}-a_{4} x^{5},
\end{aligned}
$$

where $a_{i}$ are unknown constants. In view of the boundary conditions, we have the following relations:

$$
\begin{aligned}
& 2\left(a_{2}-a_{1}\right)=-1, \\
& 2\left(a_{2}-a_{1}\right)+6\left(a_{3}-a_{2}\right)+12\left(a_{4}-a_{3}\right)-20 a_{4}=-2 e, \\
& 24\left(a_{4}-a_{3}\right)=-3, \\
& 24\left(a_{4}-a_{3}\right)-60 a_{4}=-4 e .
\end{aligned}
$$

Substituting the trial function (6) into (5), then making it stationary under the constraints of (7)-(10) with respect to $a_{i}$, we can identify all the unknown constants $a_{i}$.

\section{References}

[1] A.M. Wazwaz, The numerical solution of sixth-order boundary value problems by the modified decomposition method, Appl. Math. Comput. 118 (2001) 311-325.

[2] A.M. Wazwaz, The modified Adomian decomposition method for solving linear and nonlinear boundary value problems of 10 th-order and 12 th-order, Int. J. Nonlin. Sci. Numer. Simul. 1 (1) (2000) 17-24.

[3] J.H. He, Semi-inverse method of establishing generalized variational principles for fluid mechanics with emphasis on turbomachinery aerodynamics, Int. J. Turbo \& Jet-Engines 14 (1) (1997) 23-28. 